\title{
Research on Anti-Offset Characteristics of Static Wireless Charging for Electric Vehicles
}

\author{
Bingqian Zhang ${ }^{1, *}$, Haiyan Zhang ${ }^{1}$ \\ ${ }^{1}$ Electrical Engineering, Shanghai Dianji University, Shanghai, China
}

\begin{abstract}
The static wireless charging of electric vehicles is more convenient than traditional charging methods, but due to man-made parking will cause the coil to shift, which will cause the transmission power of the system to fluctuate and affect the stability of the system. Through the analysis of the traditional doublesided resonant system, it is found that it is more sensitive to the coupling coefficient $\mathrm{k}$ and the primary current will increase suddenly when $\mathrm{k}$ approaches zero. In order to solve these two problems, this paper compares four different resonance conditions and performs a large number of simulation experiments show that the antioffset performance of the system is the highest when both the primary and secondary sides are designed to be detuned, but a certain efficiency is sacrificed. When the bilateral detuned system is in the range of 2.4 times the coupling coefficient $\mathrm{k}$, then the fluctuation of transmission power is less than $15 \%$, and the maximum transmission efficiency is $58 \%$. It has good anti-offset ability. And when k approaches zero, the primary current will not increase suddenly. The security performance of the system is greatly improved.
\end{abstract}

\section{INTRODUCTION}

With the advent of the new era, new energy Electric Vehicles (EV) have entered people's field of vision. Traditional electric vehicle charging is wired contact charging. This wired charging method has many disadvantages. For example, the high frequency of plug-in charging can easily cause problems such as equipment aging and wearing. At the end of the 19th century, American physicist Nikola Tesla built the first Wireless Power Transfer (WPT) test system, from which the world began to study the technology related to WPT. This technology has been applied to mobile mechanical and electrical equipment, electric vehicles, underwater equipment ${ }^{[1]}$ and other fields. In practical applications, it is found that the deviation of the position of the receiving coil will cause the transmission parameters such as transmission power and efficiency to change. Due to manmade parking, it is easy to cause the longitudinal and lateral deviation of the coil and the vehicle vibration to cause a certain angular deviation of the chassis, which leads to fluctuations in the transmission power of the system and affects the stability of the system. A typical WPT charging system for electric vehicles is shown in figure $1^{[2]}$.

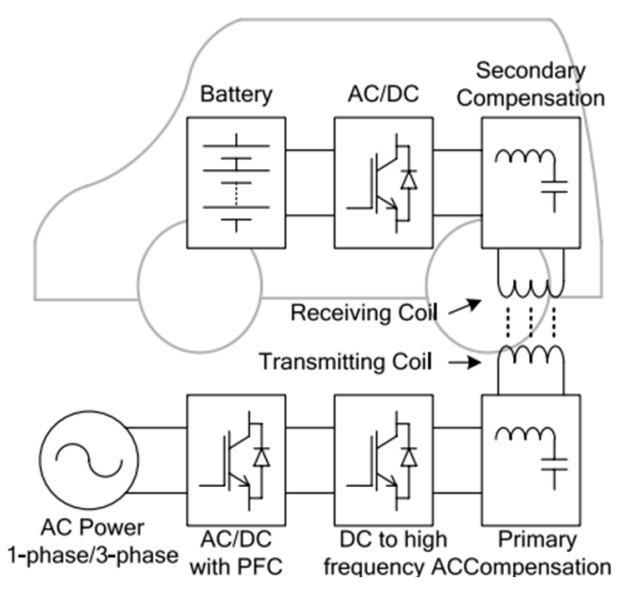

Fig. 1. Typical EV WPT charging system.

Currently, there are two main research points to improve the anti-offset capability of EV WPT: the magnetic coupling structure of the system and the compensation network. Literature [3] is that the team of Professor Boys JT of the University of Auckland proposed a flux pipe in 2010, which is a new topology applied to EVs. This topology can better improve the magnetic flux path. It is verified that its anti-offset ability is better than circular topology. In reference [4], the team proposed a double coil structure-DD type coil in 2013. This coil combines the advantages of a circular coil and a Flux pipe type coil. The magnetic flux path length of this coil is approximately twice that of the circular coil. Another way to improve the anti-offset performance of EV WPT is to use a suitable compensation network. Literature [5] is the team of Professor J.L. Villa of the University of Zaragoza, who proposed a serial-parallel-serial (SPS) compensation 
topology. This topology is a combination of two basic topologies, namely Series-Series (SS) and Parallel-Series (PS). Experiments show that the new topology can improve the anti-offset performance of the transmission system. In the literature [6], the research team of San Diego State University analyzed the topological structure of SS compensation and dual LCC compensation respectively, and verified them through experiments. The results show that dual LCC compensation has better performance than SS compensation. The above compensation topology can improve the anti-offset performance of the coil, but the cost and complexity of the system increase accordingly.

In the actual EV WPT system, the SS compensation topology is usually used. Generally, the transmitting and receiving side coils use full resonance to make the WPT system have better transmission performance, but according to the literature [7], when the coupling coefficient $\mathrm{k}$ changes, the fully resonant SS topology is extremely susceptible, and when $\mathrm{k}$ gradually approaches zero, the system will experience excessive current.

In order to solve the above problems, this paper proposes to apply the dual-sided non-resonant design to EV WPT after comparing four different resonance modes. The design uses non-resonance on both the primary and secondary sides of the circuit. A reasonable design of the bilateral detuning rate can improve the anti-offset performance of the system. The power fluctuation degree of the system becomes stable, which can transmit power more stably, and at the same time improve the degree of freedom of the receiving side coil.

\section{Non-resonant EV WPT system characteristics}

\subsection{EV WPT topology in non-resonant state}

The EV WPT discussed in this article is a magnetic coupling resonance type, which uses a SS basic topology. The topology in its non-resonant mode is analyzed by a mutual inductance model, the network relationship is deduced and the primary and secondary sides are designed. This lays the foundation for studying the anti-offset performance of the system.

\subsubsection{Mutual inductance model of non-resonant system}

The following figure 2 shows the main circuit of the incomplete resonance system topology. Take the doublesided detuning circuit of the SS compensation topology as an example. Both the primary and secondary sides adopt SS-type capacitor series compensation, and the compensation capacitor parameters of the primary and secondary sides are all set to non-resonant values, $U_{a}$ is the primary input DC voltage, which is changed into high frequency $\mathrm{AC}$ voltage $U_{2}$ through high frequency inverter. $S_{1}, S_{2}, S_{3}, S_{4}$ is the switch tube of the inverter part, $C_{1}$ and $L_{1}$ is the compensation capacitance of the primary side and the self-inductance of the coil, respectively. $C_{2}$ and $L_{2}$ is the compensating capacitance of the secondary side and the self-inductance of the coil, respectively, $U_{2}$ is the output $\mathrm{AC}$ voltage after secondary resonance, $D_{1}, D_{2}, D_{3}, D_{4}$ is the diode of the rectifier, $M$ is the mutual inductance between the primary and secondary coils, $U_{b}$ represents the actual battery voltage that needs to be recharged.

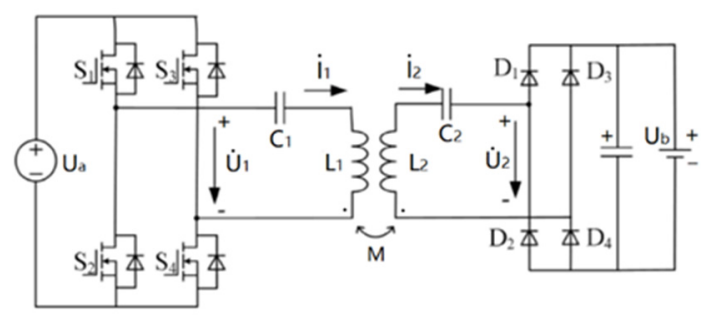

Fig.2.Non-full resonant topology main circuit.

In order to simplify the calculation, the primary and secondary compensation capacitors and the parasitic resistors of the coils on both sides of the main circuit need to be ignored, and the simplified mutual inductance circuit model is shown in figure 3 .

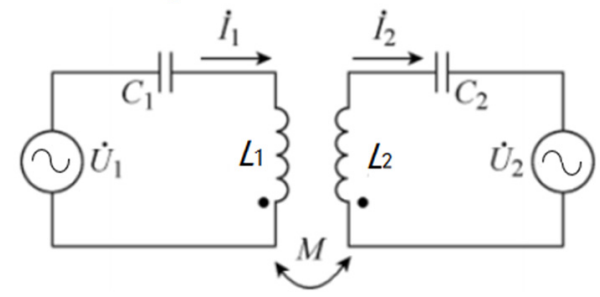

Fig. 3. Typical EV WPT charging system.

In figure 3 , the expression of $\dot{U}_{1}$ and $\dot{U}_{2}$ is as follows:

$$
\begin{aligned}
& \left|\dot{U}_{1}\right|=\frac{2 \sqrt{2}\left|U_{a}\right|}{\pi} \\
& \left|\dot{U}_{2}\right|=\frac{2 \sqrt{2}\left|U_{b}\right|}{\pi}
\end{aligned}
$$

Write the KVL equation for figure 3 as follows:

$$
\begin{aligned}
& \dot{U}_{1}=\dot{I}_{1} Z_{1}-\dot{I}_{2} \cdot j \omega M \\
& \dot{U}_{2}=\dot{I}_{1} \cdot j \omega M-\dot{I_{2}} \cdot Z_{2}
\end{aligned}
$$

Among them, $Z_{1}=j \omega L_{1}+1 /\left(j \omega C_{1}\right), M=k \sqrt{L_{1} L_{2}}$, $Z_{2}=j \omega L_{2}+1 /\left(j \omega C_{2}\right), \omega$ is the switching angle frequency, $k$ is the coupling coefficient of the primary and secondary coils.

The EV WPT in this article adopts a two-sided detuning design, so it is necessary to introduce a detuning rate to indicate the specific degree of detuning. The detuning rate is defined as: 


$$
\gamma=1-\left|\frac{Z_{C}}{Z_{L}}\right|
$$

Among it, $Z_{L}=j \omega L, Z_{C}=1 /(j \omega C)$.

\subsubsection{Parameter design of non-resonant system}

The anti-offset of the EV WPT system is the ability of the system to transmit power stably even after the coil is offset. The less sensitive the transmission power of the system is to the change of mutual inductance and the smaller the degree of power fluctuation, the stronger the anti-offset performance of WPT. The parameter design of the nonresonant system needs to have as little power fluctuation as possible within the set $\mathrm{k}$ range.

When the coupling coefficient fluctuates within the set range $k_{\min } \sim k_{\max }$, The expression of the system selfinductance is

$$
L=\frac{\left|\dot{U}_{1}\right|\left|\dot{U}_{2}\right|}{2 \omega P_{\max s}} \sqrt{\frac{1+\beta^{2}}{\beta^{2} k_{\min }^{2}}}
$$

In the formula:

$$
\beta=\frac{k_{\max }}{k_{\text {min }}}
$$

In the actual magnetic coupling resonance system, The value of $k_{\min }$ and $\beta$ are given. The self-inductance values of the primary and secondary sides of the system can be solved by combining equations (1), (2), and (6). It should be noted that the non-resonant detuning rate of the primary side requires $\gamma>0$, At this time, the capacitance is under-compensated, which can make the resonance part of the primary side inductive, and the system is easy to realize soft switching. When designing the parameters of capacitance compensation for the non-resonance of the secondary side, $\gamma<0$ is needed. That is, the capacitor is overcompensated in order to realize soft switching. The compensation capacitance of the non-resonant system can be solved according to the formula (8):

$$
C=\frac{1}{4(1-\gamma) \pi^{2} f^{2} L}
$$

In this paper, the positive and negative series measurement method is used to measure the mutual inductance when the coil is offset, and the expression of mutual inductance is as follows:

$$
M=\frac{L_{11}-L_{22}}{4}
$$

Where $L_{11}$ is the total inductance of the primary and secondary coils in forward series, and $L_{22}$ is the total inductance in reverse series.

\subsection{Parameter design of compensation network for non-resonant system}

\subsubsection{Primary side compensation network}

The experimental frequency of this paper is $f=90 \mathrm{kHz}$. The switch angular frequency is

$$
\omega=2 \pi f=565200 \mathrm{rad} / \mathrm{s}
$$

The maximum transmission power designed by the system is $P_{\max s}=80 \mathrm{~W}$, Minimum value of coupling coefficient is $k_{\min }=0.1, \beta=2$. The DC voltage input by the system is $U_{a}=48 \mathrm{~V}$. Battery voltage on the secondary side is $U_{b}=20 \mathrm{~V}$. In order to facilitate the calculation, the primary and secondary coils use the same parameter $L_{1}=L_{2}=L$, then the actual self-inductance of the coil is as follows:

$$
L=\frac{\left|\dot{U}_{1}\right|\left|\dot{U}_{2}\right|}{2 \omega P_{\max s}} \sqrt{\frac{1+\beta^{2}}{\beta^{2} k_{\text {min }}^{2}}}=96 \mu \mathrm{H}
$$

When the primary side resonates, the capacitance of the primary side is

$$
C_{1}=\frac{1}{\omega^{2} L_{1}}=32.6 n F
$$

When the non-resonant design is carried out on the primary side, the detuning rate $\gamma$ should be considered. Different $\gamma$ can be obtained from equation (5), which will make the compensation parameters of the capacitor different. And the original side compensation capacitance value should be solved according to formula (8), it should be noted that the detuning rate needs to be greater than zero to ensure that the inverter can achieve soft switching. The values of the coil inductance and the primary compensation capacitor when the detuning ratio takes different values are shown in table 1 .

Table 1. Value of the primary compensation capacitor.

\begin{tabular}{|c|c|c|}
\hline$L_{1}$ & $\gamma$ & $C_{1}$ \\
\hline \multirow{3}{*}{$96 \mu H$} & 0 & $32.6 \mathrm{nF}$ \\
\cline { 2 - 3 } & 0.2 & $40.7 \mathrm{nF}$ \\
\cline { 2 - 3 } & 0.25 & $43.4 \mathrm{nF}$ \\
\hline
\end{tabular}

\subsubsection{Secondary side compensation network}

The parameters of the primary and secondary resonant coils designed in this paper are the same, so the compensation capacitance at the secondary side of the circuit is the same as the capacitance at the primary resonance, and its value is

$$
C_{1}=C_{2}=32.6 n F
$$

When the non-resonant design is performed on the secondary side, formula (8) is also used to solve the 
problem. The difference with the primary side nonresonant design is that the inverter can realize soft switching only when the $\gamma$ value here is less than zero.

When the detuning rate is different, the secondary side compensation capacitor and coil inductance are shown in table 2 .

Table 2. Value of secondary compensation capacitor

\begin{tabular}{|c|c|c|}
\hline$L_{2}$ & $\gamma$ & $C_{2}$ \\
\hline \multirow{3}{*}{$96 \mu H$} & 0 & $32.6 \mathrm{nF}$ \\
\cline { 2 - 3 } & -0.2 & $27.1 \mathrm{nF}$ \\
\cline { 2 - 3 } & -0.25 & $26 \mathrm{nF}$ \\
\hline
\end{tabular}

In the simulation experiment, by using the primary and secondary capacitance values of different detuning rates in table 1 and table 2, different working modes of the circuit can be realized, so that the anti-offset properties of different detuning circuits can be studied.

\section{EV WPT detuning circuit simul-ation experiment}

\subsection{Simulation circuit and parameters of non- resonant system}

In order to verify the transmission performance of the dual-side detuned EV WPT system proposed in this article, a model is built in PSIM, and the system simulation circuit model is shown in Figure 4.

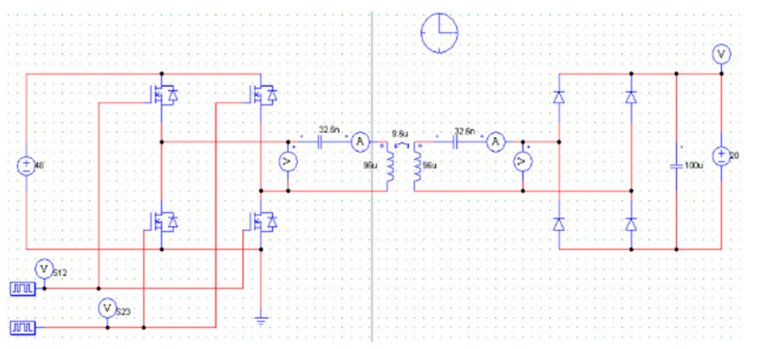

Fig. 4. System simulation circuit model.

Due to the need to study the transmission characteristics under different resonance conditions, the compensation capacitor values on both sides will change. The specific compensation capacitor values are modified in conjunction with table 1 and table 2 . Take bilateral detuning as an example, where the detuning rate is 0.2 and -0.2 respectively, and the simulation parameters of the system are shown in table 3 .

Table 3. Simulation parameters of the system.

\begin{tabular}{|c|c|}
\hline Simulation parameters & Value \\
\hline$U_{a}$ & $48 \mathrm{~V}$ \\
\hline$U_{b}$ & $20 \mathrm{~V}$ \\
\hline$f$ & $90 \mathrm{kHZ}$ \\
\hline$C$ & $100 \mu F$ \\
\hline$L_{1}$ and $L_{2}$ & $96 \mu H$ \\
\hline
\end{tabular}

\begin{tabular}{|l|l|}
\hline$C_{1}$ & $40.7 \mathrm{nF}$ \\
\hline$C_{2}$ & $27.1 \mathrm{nF}$ \\
\hline
\end{tabular}

\subsection{System current characteristics in different resonance situations}

In this paper, the actual position movement of the two coils is expressed by changing the coupling coefficient between the coils, and the primary current, transmission power and efficiency of the system are studied to analyze the anti-offset performance of the system.

The other parameters of the simulation remain unchanged, the coupling coefficient is 0.1 , and the detuning rates of the primary and secondary sides are 0.2 and -0.2 respectively. According to table 1 and table 2 , the primary and secondary side capacitance compensation values of the simulation circuit are set, and the output waveforms of the system inverter circuits under four different resonance conditions can be obtained, as shown in figure 5 below.

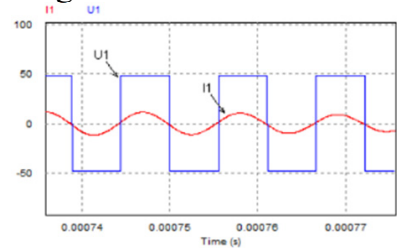

(a) Full resonance

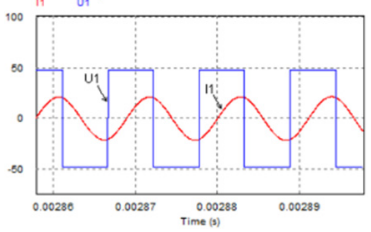

(c) Secondary side detunes

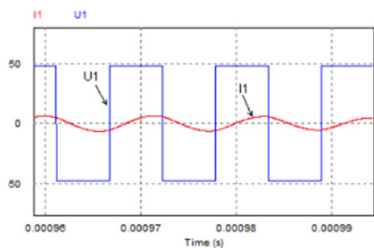

(b) Primary side detunes

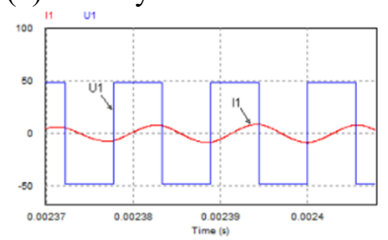

(d) Bilateral detunes
Fig. 5. Inverter output waveforms with different detuning conditions.

From the four waveforms of (a), (b), (c) and (d) in figure 5 , it can be seen that the output voltages of the inverters with full resonance, primary detuning, secondary detuning and bilateral detuning are all ahead of the output current, and soft switching can be realized.

This paper simulates the deviation of the actual coil by changing $\mathrm{k}$. By modifying the compensation capacitance values on both sides of the model for many experiments, the following four different resonance modes of the primary current and the coupling coefficient $\mathrm{k}$ can be obtained. The relationship between them is shown in Figure 6 below.

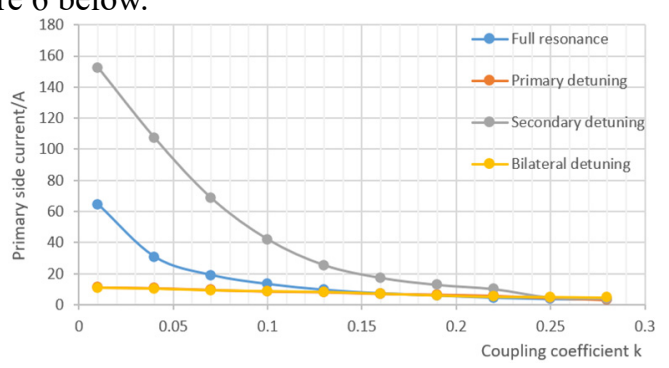

Fig. 6. Relationship between primary current and $\mathrm{k}$ in four resonance situations 
As can be seen from figure 6, when the coupling coefficient is close to zero, that is, when the distance between the two coils is too large, the current on the primary side of the two cases of full resonance and secondary detuning will increase sharply and especially when the circuit is a secondary detuning design, the primary side current increases the most, so the system will have the risk of overcurrent and safety risks. When $\mathrm{k}$ approaches zero, the current changes of primary detuning and bilateral detuning are relatively stable, there is no safety hidden danger, and the current of bilateral resonant circuit is slightly less than that of primary detuning circuit. Therefore, the detuning design in this paper does not consider the secondary detuning, and further compares the output power stability of primary detuning and bilateral detuning.

\subsection{Analysis of anti-offset characteristics of non-resonant system}

The anti-offset ability of the system means that when the two coils are offset, the output power of the system is less sensitive to the coupling coefficient between the two coils, that is, the output power curve of the system is smooth, and within a given power variation range, the larger the corresponding coupling coefficient range, the better anti-migration performance of the system.

The detuning rates of the primary and secondary sides of the system are 0.2 and -0.2 respectively, and many experiments are carried out by modifying the compensation capacitance values on both sides of the model. The relationship between the output power and $\mathrm{k}$ of the system in the case of primary detuning and bilateral detuning can be obtained as shown in figure 7 below.

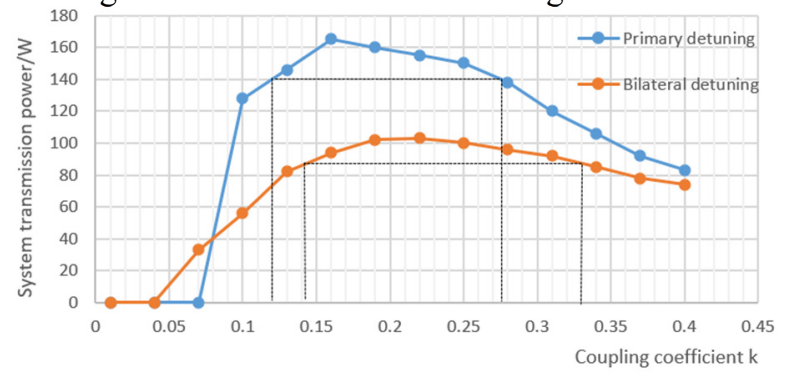

Fig. 7. Relationship between transmission power and $\mathrm{k}$

This paper is set within the range of $85 \%$ of the transmission power offset of the system, the range of the coupling coefficient of the circuit for primary detuning design is 0.12 to 0.276 , and the range of the coupling coefficient for bilateral detuning is 0.14 to 0.33 , which shows that the anti-offset ability of the system can be better improved by bilateral detuning design of the circuit.

Figure 8 is the curve of the relationship between the system efficiency of the bilateral detuned circuit and the coupling coefficient $\mathrm{k}$.

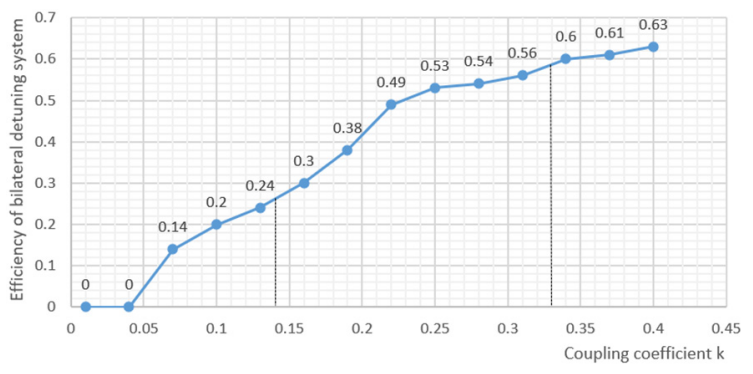

Fig. 8. The relationship between the efficiency of the two-sided detuning system and $\mathrm{k}$.

As can be seen from figure 8 , when the range of the coupling coefficient of the bilateral detuned circuit is 0.14 to 0.33 , the transmission efficiency of the system is up to $58 \%$ and the lowest is $26 \%$, and the coupling coefficient $\mathrm{k}$ is proportional to the efficiency of the bilateral detuned system. Compared with this paper, the main research is to optimize the anti-offset performance of the system, and its efficiency can be further improved by improving the structural parameters of the system.

\section{Conclusion}

For the traditional EV WPT system with bilateral resonance, when the coupling coefficient of the two coils tends to zero, the current on the primary side will suddenly increase and there is a risk of overcurrent, and it is more sensitive to the coupling coefficient. In view of these two problems, this article proposes three different detuning situations are compared and analyzed through simulation experiments. It is concluded that the anti-offset performance of the system is the highest when both the primary and secondary sides are designed for detuning, but a certain efficiency is sacrificed. When the double-sided detuning system is within the range of 2.4 times of $\mathrm{k}$, the system transmission power fluctuates within $15 \%$, and the maximum transmission efficiency reaches $58 \%$. It has good anti-offset ability, and at the same time, there is no sudden increase in the primary current when $\mathrm{k}$ tends to zero, which greatly improves the safety performance of the system.

\section{References}

1. Askari A, Stark R, Curran J, et al. Underwater wireless power transfer[C]// Wireless Power Transfer Conference. IEEE,2015.

2. Li S, Mi C C. Wireless Power Transfer for Electric Vehicle Applications[J]. Emerging \& Selected Topics in Power Electronics IEEE Journal of, 2015, 3(1):417.

3. Budhia M, Covic G, Boys J. A new IPT magnetic coupler for electric vehicle charging systems[J]. 2010:2487-2492.

4. Budhia M, Boys J T, Covic G A, et al. Development of a Single-Sided Flux Magnetic Coupler for Electric Vehicle IPT Charging Systems[J]. IEEE Transactions 
on Industrial Electronics, 2013, 60(1):318-328.

5. Juan L. Villa, Jesús Sallán, José Francisco Sanz Osorio. High-Misalignment Tolerant Compensation Topology For ICPT Systems[J]. IEEE Transactions on Industrial Electronics, 2012,59(2):945-951.

6. Li W, Zhao H, Deng J, et al. Comparison Study on SS and Double-Sided LCC Compensation Topologies for EV/PHEV Wireless Chargers[J]. IEEE Transactions on Vehicular Technology, 2016, 65(6): 4429-4439.

7. Sohn Y H, Choi B H, Lee E S, et al.General Unified Analyses of Two-Capacit-or Inductive Power Transfer Systems: E-quivalence of Current-Source SS and SP Compensations[J].IEEE Transactions on Power Electronics,2015,30(11):6030-6045. 